\title{
Laparoscopy-assisted transgastric endoscopic retrograde cholangiopancreatography in bariatric Roux-en-Y gastric bypass patients
}

Authors

Institutions
Christophe Snauwaert ${ }^{1,2}$, Pierre Laukens ${ }^{2}$, Bruno Dillemans ${ }^{2}$, Jacques Himpens ${ }^{3}$, Danny De Looze ${ }^{4}$, Pierre Henri Deprez ${ }^{1}$, Abdenor Badaoui ${ }^{5}$

Institutions are listed at the end of article. submitted 6. February 2015 accepted after revision 2. April 2015

\section{Bibliography}

DOI http://dx.doi.org/

10.1055/s-0034-1392108

Published online: 23.6.2015

Endosc Int Open 2015; 03: E458-E463

(c) Georg Thieme Verlag KG Stuttgart · New York

E-ISSN 2196-9736

\section{Corresponding author} Christophe Snauwaert, MD Dudzeelse Steenweg 149 8000 Brugge Belgium

Fax: +32-2-7648927 christophe.snauwaert@hotmail. com
Background: Performing endoscopic retrograde cholangiopancreatography in bariatric patients who underwent Roux-en-Y gastric bypass surgery is challenging due to the long anatomical route required to reach the biliopancreatic limb.

Aim: Assessment of the feasibility and performance of laparoscopy-assisted transgastric endoscopic retrograde cholangiopancreatography.

Methods: A retrospective multicenter observational consecutive-patient cohort study of all patients in the period May 2008 to September 2014 with a history of Roux-en-Y gastric bypass who presented with complicated biliary disease and who underwent a laparoscopy-assisted transgastric endoscopic retrograde cholangiopancreatography. The laparoscopy-assisted transgastric endoscopic retrograde cholangiopancreatography procedure was similar in all centers and was performed through a $15 \mathrm{~mm}$ or $18 \mathrm{~mm}$ trocar that was inserted in the gastric remnant. Cholecystectomy was performed concomitantly when indicated.

Results: In total, 23 patients underwent a laparoscopy-assisted transgastric endoscopic retrograde cholangiopancreatography procedure. Two pa-

\section{Introduction}

$\nabla$

Rapid weight loss after Roux-en-Y gastric bypass (RYGB) is a risk factor for the development of biliary complications, particularly cholelithiasis, with subsequent risk of choledocholithiasis and gallstone pancreatitis. It has been suggested that concomitant cholecystectomy (CCE) in obese patients undergoing RYGB may reduce future gallstonerelated morbidity. However, to date, concomitant CCE is not recommended in obese patients undergoing RYGB since only a minority (about $7 \%$ to $8 \%$ ) will become symptomatic and require delayed CCE, which would expose many patients to an unnecessary procedure [1]. In turn, endoscopic access to the biliary tree in post-RYGB patients tients required a mini-laparotomy for transgastric access because of a complex surgical history resulting in multiple adhesions. Indications included ascending cholangitis, choledocholithiasis, and biliary pancreatitis. Of the 23 patients, 13 underwent concomitant cholecystectomy. All patients successfully underwent biliary cannulation and sphincterotomy. No endoscopic procedure-related complications (i.e. bleeding, pancreatitis or retroperitoneal perforation) occurred. Mean hospital stay was 2.8 days (range $2-4$ ).

Conclusions: Transgastric endoscopic retrograde cholangiopancreatography is a feasible approach in the treatment of pancreaticobiliary disease in Roux-en-Y gastric bypass patients, without major complications in our series and allows endoscopic treatment and cholecystectomy to be performed consecutively in a single procedure. In Roux-en-Y gastric bypass patients without a history of prior cholecystectomy presenting with complicated gallstone disease, combining cholecystectomy and transgastric endoscopic retrograde cholangiopancreatography as a first-line approach may be a valid treatment strategy.

with biliopancreatic disorders is technically challenging due to specific anatomical rearrangements ( $\bullet$ Fig. 1 ). To perform endoscopic retrograde cholangiopancreatography (ERCP) in these patients, access to the pancreaticobiliary limb is necessary. This anatomical rearrangement prevents passage to the major papilla with a conventional side-viewing duodenoscope.

One option is to perform a balloon enteroscopyassisted ERCP (BEA-ERCP) via the gastric pouch and the Roux (alimentary) limb up the biliopancreatic limb to the major papilla. However, the estimated success rate (i.e. biliary cannulation) of ERCPs performed in long-limb surgical bypass patients is approximately $60 \%$ in most of the literature [2-9]. 


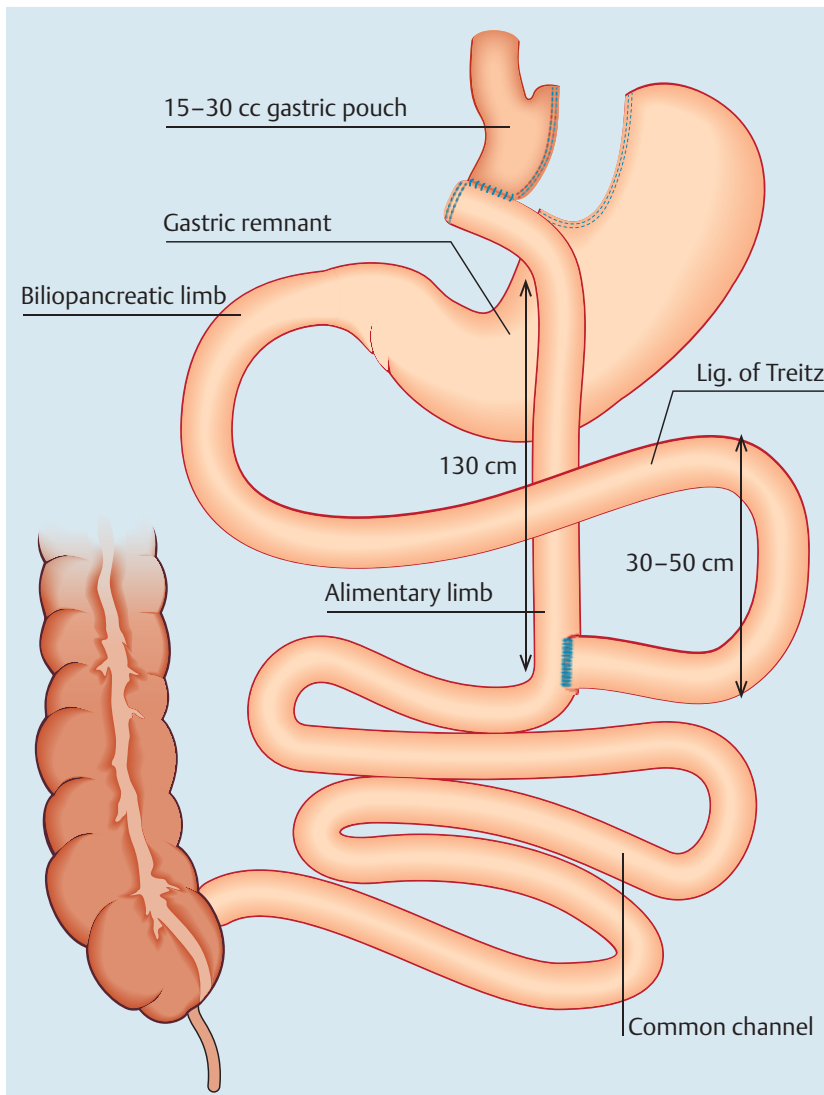

Fig. 1 Roux-en-Y gastric bypass configuration. The proximal jejunum is divided distal to the ligament of Treitz. A side-to-side jejunojejunostomy is performed. The gastric pouch is created by repeated application of a linear cutter. The pouch is based on the lesser curve and oriented vertically with exclusion of the gastric fundus. The Roux limb is brought up in a retrocolic position and lies anterior to the stomach remnant and a gastrojejunostomy is performed. The length of the Roux limb is usually $\pm 130 \mathrm{~cm}$ and the length of the biliopancreatic limb from the ligament of Treitz to the jejunojejunal anastomosis usually varies from 30 to $50 \mathrm{~cm}$.

Laparoscopy-assisted transgastric ERCP (LA-ERCP) in contrast, has been shown to be superior to BEA-ERCP in terms of therapeutic success rate (biliary cannulation rates up to $100 \%$ have been described) and is associated with a significantly shorter endoscopic procedure time, but not a shorter total procedure time (=laparoscopy+ERCP) $[2,4,5]$.

The aim of this retrospective study is to assess the feasibility and performance of LA-ERCP after RYGB with or without combined CCE.

\section{Patients/material and methods}

\section{$\nabla$}

\section{Methods}

We analyzed retrospectively the data from all bariatric RYGB patients who underwent a LA-ERCP in the study centers from May 2008 to September 2014. Informed consent was obtained from all patients according to usual clinical practice. Patients were identified using an electronic database that captured all endoscopic procedures performed at each center. Clinical data including age, gender, preoperative indication, and diagnostic results were recorded in the electronic patient file.

\section{LA-ERCP procedure}

The LA-ERCP technique was similar in all cases. Standard laparoscopic access to the abdominal cavity was accomplished with insertion of four trocars. When indicated, CCE was performed. The standard LA-ERCP steps are shown in $\bullet$ Fig. 2 and $\bullet$ Video 1. First of all, mobilization of the greater curve of the antrum was achieved. A gastrotomy and purse-string suture were fashioned on the anterior side of the greater curvature of the gastric remnant near the antrum. An additional $15 \mathrm{~mm}$ or $18 \mathrm{~mm}$ trocar (Endopath ${ }^{\circledR} \mathrm{Xcel}^{\circledR}$, Ethicon, Johnson \& Johnson Medical, Diegem, Belgium) was placed in the left upper quadrant and inserted into the gastrotomy in the center of the purse-string suture. The pursestring suture was tightened and the gastric remnant was lifted up to the anterior abdominal wall. The biliopancreatic limb was occluded with an intestinal clamp to prevent "over-insufflation" of the small bowel which obscures perioperative visualization. Finally, a side-viewing endoscope (TJF 160 R, distal end outer diameter of $13.5 \mathrm{~mm}$, Olympus Corp, Tokyo, Japan) was introduced through the $15 \mathrm{~mm}$ or $18 \mathrm{~mm}$ trocar secured into the gastrotomy, and ERCP was performed under fluoroscopic guidance. Carbon dioxide gas, which has been demonstrated to be safer and superior to conventional room air insufflation, was used during endoscopic insufflation in all procedures. After removal of the scope and the $15 \mathrm{~mm}$ trocar, the purse-string was tied and the gastrotomy incision was sutured.

\section{Results}

$\nabla$

Demographic and clinical data for the 23 RYGB patients who underwent LA-ERCP are shown in $\bullet$ Table 1. A total of 23 patients were identified (median age 54 years, range 26-79). In total, 21 patients were operated laparoscopically. Laparoscopic access was converted to a mini-laparotomy in two patients due to severe adhesions. Ten patients had history of prior CCE. The median body mass index (BMI) of the study population was 26.5 (range 19.547). Indications included ascending cholangitis (all with concomitant choledocholithiasis), radiologically proven choledocholithiasis, cholecystitis combined with choledocholithiasis and cholangitis, recurrent biliary pancreatitis, cholestasis/jaundice, and a combination of a typical pain syndrome, cholelithiasis, elevated liver enzymes, and a dilated common bile duct (CBD) on ultrasound. In total, 13 patients underwent concomitant CCE, eight before and five after ERCP completion. In the majority of cases, choledocholithiasis was demonstrated (17/23).

The remaining ERCP findings of the other six patients included dilated CBD, papillary stenosis, transection of the CBD, and biliary sludge. In the first patient in whom only a dilated CBD could be established $( \pm 12 \mathrm{~mm}$ on peroperative cholangiography), a sphincterotomy was performed because of preoperative documented biochemical cholestasis (gamma-glutamyl transferase (GGT) 2027 IU/L [normal range 9-36], alkaline phosphatase [AP] $599 \mathrm{IU} / \mathrm{L}$ [normal range 40 -129]), presence of cholecystolithiasis, and a suspicion of choledocholithiasis on a preoperatively performed magnetic resonance cholangiopancreatography (MRCP). In the second patient, a sphincterotomy was performed because of well-documented cholecystolithiasis, a history of biliary colic pain episodes, and liver test abnormalities (aspartate aminotransferase [AST] level 202 IU/L [normal:<30IU/L], alanine aminotransferase [ALT] level 485IU/L [normal:<36IU/L], GGT $130 \mathrm{IU} / \mathrm{L}, \mathrm{AP} 167 \mathrm{IU} / \mathrm{L})$; the CBD appeared slightly dilated on peroperative cholangiogram $( \pm 10 \mathrm{~mm})$. In the third patient, after 

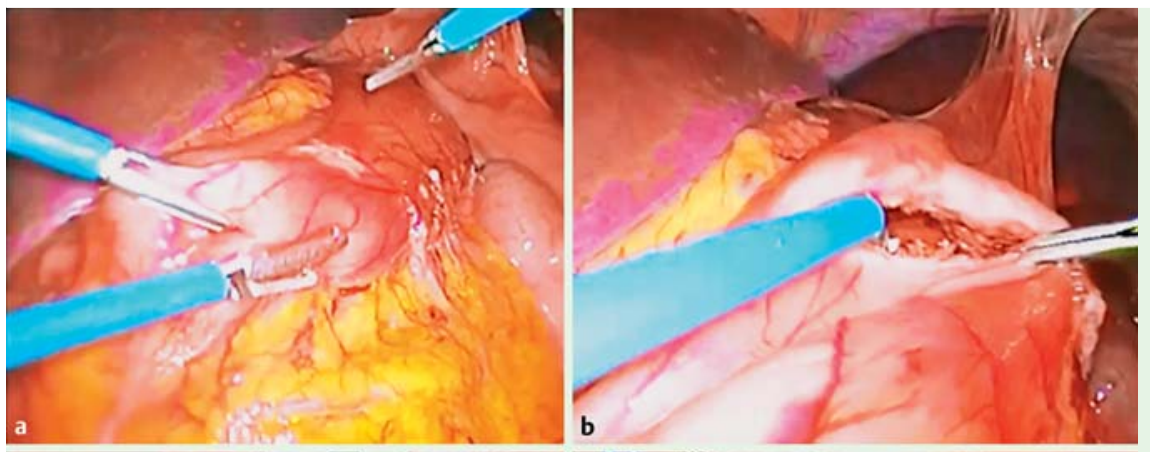

Fig. 2 Standard laparoscopy-assisted transgastric endoscopic retrograde cholangiopancreatography (LA-ERCP) steps. a, b Formation of a gastrotomy on the anterior side of the greater curvature of the gastric remnant near the antrum; $\mathbf{c}$ insertion of a $15 \mathrm{~mm}$ trocar into the gastric remnant through the gastrotomy; $\mathbf{d}$ the trocar is secured with a pursestring suture; $e, f$ ERCP with sphincterotomy and stone extraction; $\mathbf{g}$ suture of the gastrotomy incision.



arising from post-procedure fibrosis and scarring, 4 years after

Video 1

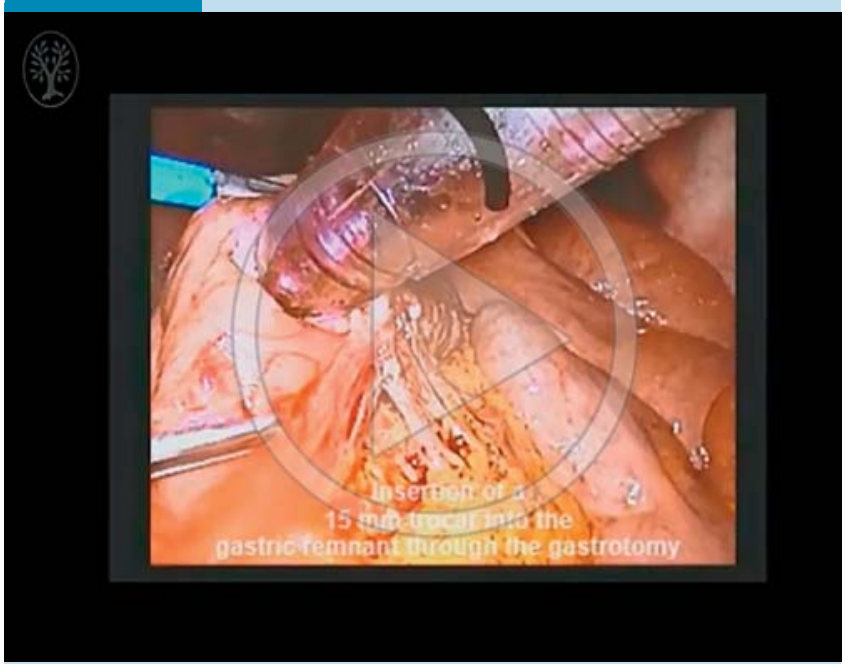

Compilation of the different consecutive steps of the laparoscopy-assisted transgastric endoscopic retrograde cholangiopancreatography (LA-ERCP) procedure.

sphincterotomy was performed, evacuation of small sludge fragments was seen, without obvious CBD stones. This patient had a recent history of cholangitis and mild pancreatitis (total bilirubin $1.71 \mathrm{mg} / \mathrm{dL}$ [normal: 0.3-1.20], AST 658 IU/L, ALT 640 IU/L, AP $152 \mathrm{IU} / \mathrm{L}$, GGT $393 \mathrm{IU} / \mathrm{L}$, lipase $595 \mathrm{IU} / \mathrm{L}[<60 \mathrm{IU} / \mathrm{L}])$. A fourth patient developed biliary pain episodes with concomitant cholestasis and cytolysis. This patient had developed a papillary stenosis previous endoscopic sphincterotomy. Endoscopic therapy was performed (new sphincterotomy) after which the patient became symptom-free and liver test abnormalities normalized. A fifth patient presented with abdominal pain and jaundice 1 week after a combined surgical procedure (RYGB and CCE) was performed. Laboratory work-up revealed a frank cholestasis (total bilirubin $4.75 \mathrm{mg} / \mathrm{dL}$, AST $133 \mathrm{IU} / \mathrm{L}$, ALT $215 \mathrm{IU} / \mathrm{L}$, GGT $178 \mathrm{IU} / \mathrm{L}$, AP $378 \mathrm{IU} / \mathrm{L})$. CT imaging showed dilatation of the intrahepatic bile ducts and the hepatic hilar region and ascites. A decision was made to perform a laparoscopy-assisted transgastric exploration of the CBD. ERCP confirmed a transection of the CBD with a large bile collection in the gallbladder bed. After external drainage, the patient eventually underwent hepaticojejunostomy. The sixth patient presented with cholestasis 5 years after a RYGB and CCE was performed. Preoperative imaging showed a dilated $\mathrm{CBD}$ without obvious $\mathrm{CBD}$ stones. At ERCP, the papilla was located adjacent to a diverticulum. Cannulation was rather difficult and use of the guidewire as a pathfinder was necessary to allow passage through a siphon at the prepapillary bile duct. The CBD appeared slightly dilated at cholangiography $( \pm 12 \mathrm{~mm})$ and after sphincterotomy, balloon sweeping of the CBD revealed small sludge fragments without obvious large CBD stones.

All patients successfully underwent biliary cannulation and sphincterotomy (in one patient, a rendezvous technique was used in which the surgeon inserted a guidewire through the cystic duct). Endoscopic procedure time was available in 19 patients. The mean endoscopic procedure time was 40.6 minutes (range 13-120). There were no real difficulties in manipulating the 
Table 1 Demographic and clinical data for the 23 Roux-en-Y gastric bypass (RYGB) patients who underwent laparoscopy-assisted transgastric endoscopic retrograde cholangiopancreatography (LA-ERCP).

\begin{tabular}{|l|l|}
\hline Patients (n=23) & \\
\hline Median age (range), years & $54(26-79)$ \\
\hline Male/female ratio & $5 / 18$ \\
\hline Indications & \\
\hline Choledocholithiasis & 4 \\
\hline Choledocholithiasis/biliary pancreatitis & 1 \\
\hline Choledocholithiasis/cholangitis & 3 \\
\hline Choledocholithiasis/pancreatitis/cholangitis & 3 \\
\hline Choledocholithiasis/cholecystitis & 1 \\
\hline Choledocholithiasis/biliary pain syndrome & 4 \\
\hline Biliary pain syndrome & 4 \\
\hline Jaundice/cholestasis & 3 \\
\hline Timing of CCE & \\
\hline Concomitant CCE & 13 \\
\hline Prior CCE & 10 \\
\hline Surgical access & \\
\hline Laparoscopy & 21 \\
\hline Laparoscopy and conversion to laparotomy & 2 \\
\hline ERCP findings & \\
\hline Choledocholithiasis & 17 \\
\hline Sludge & 1 \\
\hline Dilated CBD & 2 \\
\hline Transection of the CBD & 1 \\
\hline Papillary stenosis & 1 \\
\hline Dilated CBD / parapapillary diverticulum / sludge & 1 \\
\hline Procedural course & 23 \\
\hline Sphincterotomy & 23 (one rendezvous) \\
\hline Biliary cannulation & 17 \\
\hline Complete stone extraction & $40.6(13-120)$ \\
\hline Mean endoscopic procedure time (range), minutes & \\
\hline Post-procedural course & $2.8(2-4)$ \\
\hline Complications & \\
\hline Gastrointestinal bleeding & \\
\hline Pancreatitis & \\
\hline Perforation & \\
\hline Mean hospital stay (range), days & \\
\hline CD. & \\
\hline
\end{tabular}

CBD, common bile duct; CCE, cholecystectomy; ERCP, endoscopic retrograde cholangiopancreatography.

scopes in the patients who were still obese. No ERCP-related complications (bleeding/(retroperitoneal) perforation/pancreatitis) occurred. There was no need for surgical re-intervention. Mean hospital stay was 2.8 days (range $2-4$ ).

\section{Discussion}

In bariatric RYGB patients, ERCP is challenging due to the complex long-length anatomy ( $\bullet$ Fig. 1) and the lack of long-length side-viewing endoscopes.

Several techniques have been described to obtain biliary access after RYGB, including minimally invasive techniques with endoscopic ultrasound (EUS)-guided and fluoroscopic guided insertion of a gastrostomy tube into the gastric remnant. In these cases, ERCP is performed percutaneously in a two-stage manner (after balloon or bougie dilatation of the matured gastrotomy tract). Transgastric ERCP can also be performed in a one-stage manner in the case of EUS-guided or enteroscopy-assisted sutured gastropexy [10-12]. However, extensive discussion of these particular techniques is beyond the scope of this article.
BEA-ERCP for the treatment of biliopancreatic disorders in bariatric RYGB patients is feasible, but, even in experienced hands, the average success rate is approximately $60 \%$ [2-9]. Disadvantages of this technique are its long procedural time (associated with a delay in identifying the enteroenterostomy site) and the difficult orientation of the endoscopic equipment. The papilla is usually reached from a different angle as with a duodenoscope which results in different and more difficult approaches for biliary cannulation. Enteroscopes also lack an elevator which means scope positioning has to be precise to maneuver the endoscopic instruments into the correct orientation. Another disadvantage of the BEA-ERCP technique is the limited availability of reliable accessories required for performing ERCP $[2,9]$. The anatomical RYGB construction and more specifically the "total limb length" (Roux limb + biliopancreatic limb from the ligament of Treitz to the jejunojejunal anastomosis) is considered to be a limiting factor for therapeutic success of BEA-ERCP $(-$ Fig. 1$)$. A long limb bypass may prevent access to the major papilla through use of the BEAERCP technique. Schreiner et al. reported a significantly higher rate of therapeutic success in patients having a total limb length less than $150 \mathrm{~cm}$ compared with those with a total limb length of $150 \mathrm{~cm}$ or longer ( $88 \%$ vs. $25 \%$, respectively) [2]. However, a total limb length of at least $150 \mathrm{~cm}$ is standard in gastric bypass surgery nowadays (the standard gastric bypass procedure involves a Roux limb length of 100 to $150 \mathrm{~cm}$ ) [13]. Furthermore, the majority of our patients (13/23) also required CCE (and consequently laparoscopic exploration). The LA-ERCP technique allowed ERCP and CCE to be performed consecutively in a single procedure in these patients without any complications, which, to date, has only been case reported.

When taking into account the cost of failed initial BEA-ERCP in the majority of patients with a "total limb length" of $>150 \mathrm{~cm}$, and the fact that nowadays, many RYGB patients who require ERCP also require CCE, LA-ERCP and combined CCE as a first-line therapy may result in less need for further procedures, equivalent morbidity and hospital stay, technically less demanding ERCP procedures with decreased endoscopist time, and decreased total cost.

\section{Technical and practical considerations and pitfalls of LA-ERCP}

In general, endoscopic sphincterotomy is performed in the treatment of choledocholithiasis, which was the case in the majority of our patients (17/23). Then again, after RYGB, ERCP is not only a therapeutic but also a diagnostic procedure since EUS (e.g. to exclude small distal CBD stones which can easily be missed on MRCP) cannot be performed preoperatively and the decision to conduct LA-ERCP and sphincterotomy cannot rely on EUS.Of course, the same problem concerning diagnosis is observed when performing BEA-ERCP. On the other hand, RYGB patients with sphincter of Oddi dysfunction or papillary stenosis can present with similar symptoms and biochemical abnormalities. Performing endoscopic sphincterotomy can pose an increased risk of post-ERCP pancreatitis in these cases. However, when choledocholithiasis is suspected preoperatively but not observed preoperatively, it is often difficult not to perform endoscopic papillotomy given the efforts that have been made to obtain access to the papilla.

Regarding the technical difficulty of the procedure, LA-ERCP has several advantages in contrast to BEA-ERCP, since ERCP can be performed with a standard side-viewing endoscope through a minimally invasive access to the stomach $[2,14]$. The results of 
our patient cohort confirm the data from prior studies and show that the laparoscopy-assisted transgastric ERCP technique allows reliable access to the duodenum using standard side-viewing duodenoscopes. However, some technical aspects and pitfalls have also to be considered in this technique. Insufflation of carbon dioxide gas is advised during the endoscopic procedure, which has been demonstrated to be safer and superior to conventional room air endoscopic insufflation in the case of intraperitoneal gas leakage. Concomitant CCE after completion of the ERCP can be rendered more difficult due to bowel distension, which can be prevented by application of an intestinal clamp proximal to the angle of Treitz. Dissection and identification of the cystic duct, but suspending definitive CCE until ERCP is performed, is considered to be the safest option. This permits a less complicated insertion of a guidewire into the cystic duct when CBD access is difficult (rendezvous technique), which was necessary in 1/23 patients. However, there was no real preference with regard to the exact timing of concomitant CCE pre- or post-ERCP, which primarily depended on technical and logistical considerations and did not appear to influence the performance of both procedures $[5,16]$. The purse-string on the greater curvature of the antrum of the gastric remnant has to be tightly fixed around the trocar to prevent loss of insufflation pressure. The gastrotomy should be made as lateral as possible along the greater curvature to permit smooth intubation of the pylorus. A $15 \mathrm{~mm}$ or $18 \mathrm{~mm}$ laparoscopic trocar is used to allow gentle maneuvering of the duodenoscope into the gastric remnant [15-17]. However, careless retraction of the endoscope in an angulated position can result in "peeling" of the coating of the duodenoscope ( $\bullet$ Fig.3). Furthermore, there are some differences to standard biliary cannulation in performing LA-ERCP. Straightening of the scope is not possible and the major papilla has to be approached from a greater distance, making cannulation and performing sphincterotomy suboptimal. Therefore, to facilitate biliary cannulation, the $15 \mathrm{~mm}$ trocar needs to be oriented towards the pylorus, which usually requires an assistant to maintain torque and change positions.

We did not encounter any significant intraoperative complications due to the laparoscopic procedure itself. However, performance of the LA-ERCP technique is influenced by the presence of postoperative adhesions, which renders the transgastric access more difficult. This is to be expected in the case of a history of open surgery (laparotomy), multiple laparoscopic interventions (with or without mesh hernia repair) and previous peritonitis or abscess formation. In 2/23 patients, transgastric access could only be achieved by mini-laparotomy because of complex surgical history (laparotomy, small-bowel resections, abdominal wall abscess, etc) resulting in the formation of multiple adhesions. Concerning postoperative complications, we could identify one case of wound infection which was successfully treated with oral antibiotics. None of the patients in the study population required surgical re-intervention. Postoperative care was rather similar to conventional CCE or other laparoscopic procedures. All patients received a subhepatic Easy-Flow drainage which was removed in all patients on postoperative day 1 without evidence of bile leakage. Ryle tubes or any other nasogastric tubes were not routinely used postoperatively.

A particular disadvantage of the LA-ERCP technique is the requirement for a repeat hybrid laparo-endoscopic access to the upper quadrant when repeat ERCP is required, for instance, to manage post-sphincterotomy bleeding or residual CBD stones. When the need for repeat ERCP is anticipated during the first LA-ERCP procedure, a gastrostomy tube can be inserted through

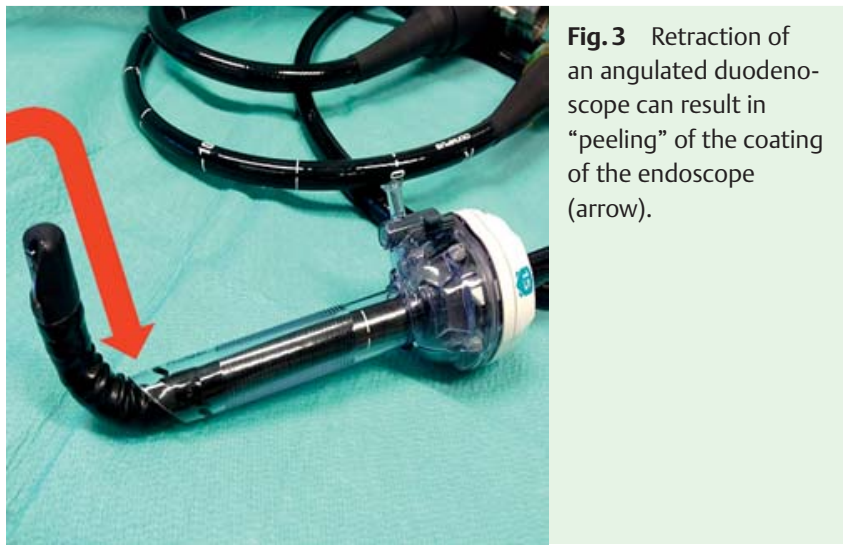

the gastrotomy incision into the lumen of the stomach. Repeat ERCP can be performed percutaneously after the surgical gastrostomy tract has matured (and usually after balloon or bougie dilatation of the gastrostomy tract) [18]. Fortunately, in our series, repeat access to the biliary tract was not necessary. In addition, none of our patients required prophylactic placement of a pancreatic stent to prevent post-ERCP pancreatitis. However, when indicated, prophylactic stenting with a $3 \mathrm{Fr}$ stent is proposed, which generally will pass spontaneously [4].

To conclude, in this hybrid laparo-endoscopic technique, close collaboration is necessary between surgeons and endoscopists, first, to harmonize the agendas of both the endoscopist and the laparoscopic surgeon and second, to ensure correct positioning and orientation of the laparoscopic, endoscopic and radiological equipment during the procedure.

\section{Conclusion}

This retrospective patient cohort clearly showed that performing laparoscopy-assisted transgastric ERCP through a $15 \mathrm{~mm}$ or 18 $\mathrm{mm}$ trocar inserted into the gastric remnant is a feasible approach in the treatment of biliopancreatic disorders in bariatric RYGB patients. LA-ERCP can provide an effective biliary access after RYGB: it is safe and reliable for the management of complicated biliary disease and can be performed by a minimally invasive approach. In our series, the LA-ERCP technique also allowed ERCP and CCE to be performed consecutively in a single procedure in all patients who required CCE, which, to date, has only been case reported. Hence, in bariatric RYGB patients without prior CCE and with complicated gallstone disease (e.g. choledocholithiasis, ascending cholangitis, etc), combining CCE and LAERCP as a first-line approach may be a valid treatment strategy and may reduce total costs because the two procedures are performed in the same intervention.

\section{Competing interests: None}

\author{
Institutions \\ ${ }^{1}$ Cliniques Universitaires Saint-Luc, Brussels, Belgium \\ 2 AZ Sint-Jan Hospital Brugge-Oostende, Bruges, Belgium \\ ${ }^{3}$ AZ Sint-Blasius General Hospital, Dendermonde, Belgium \\ ${ }^{4}$ Ghent University Hospital, Ghent, Belgium \\ ${ }^{5}$ Mont-Godinne University Hospital, Yvoir, Belgium
}




\section{Acknowledgments}

I (Christophe Snauwaert) would like to thank Dr. Pierre Laukens for his continuing mentorship and for teaching me the first steps of ERCP and therapeutic endoscopy in general. He has now retired and I wish him and his wife the best of luck for the future.

\section{References}

1 D'Hondt M, Sergeant G, Deylgat B et al. Prophylactic cholecystectomy, a mandatory step in morbidly obese patients undergoing laparoscopic Roux-en-Y gastric bypass? J Gastrointest Surg 2011; 15: 1532-1536

2 Schreiner MA, Chang L, Gluck $M$ et al. Laparoscopy-assisted versus balloon enteroscopy-assisted ERCP in bariatric post-Roux-en-Y gastric bypass patients. Gastrointest Endosc 2012; 75: 748 - 756

3 Aabakken L. Endoscopic retrograde cholangiopancreatography. Gastrointest Endosc 2012; 76: 516-520

4 Lopes TL, Clements RH, Wilcox CM. Laparoscopy-assisted ERCP: experience of a high-volume bariatric surgery center (with video). Gastrointest Endosc 2009; 70: 1254-1259

5 Bertin PM1, Singh K, Arregui ME. Laparoscopic transgastric endoscopic retrograde cholangiopancreatography (ERCP) after gastric bypass: case series and a description of technique. Surg Endosc 2011; 25: $2592-2596$

6 Gutierrez JM, Lederer H, Krook JC et al. Surgical gastrostomy for pancreatobiliary and duodenal access following Roux en Y Gastric bypass. J Gastrointest Surg 2009; 13: 2170-2175

7 Emmett DS, Mallat DB. Double-balloon ERCP in patients who have undergone Roux-en-Y surgery: a case series. Gastrointest Endosc 2007; 66: $1038-1041$

8 Skinner M, Popa D, Neumann $H$ et al. ERCP with the overtube-assisted enteroscopy technique: a systematic review. Endoscopy 2014; 46: $560-572$

9 Moreels TG. Altered anatomy: enteroscopy and ERCP procedure. Best Pract Res Clin Gastroenterol 2012; 26: 347-357
10 Attam R, Leslie D, Freeman $M$ et al. EUS-assisted, fluoroscopically guided gastrostomy tube placement in patients with Roux-en-Y gastric bypass: a novel technique for access to the gastric remnant. Gastrointest Endosc 2011; 74: 677-682

11 Attam R, Leslie D, Arain MA et al. EUS-guided sutured gastropexy for transgastric ERCP (ESTER) in patients with Roux-en-Y gastric bypass: a novel, single-session, minimally invasive approach [article in German]. Endoscopy 2015: [Epub ahead of print]

12 Attam $R$, Leventhal TM, Trikudanathan $G$ et al. Single-session, transgastric ERCP using sutured gastropexy for treatment of pancreatic duct leak in a patient with Roux-en-Y gastric bypass anatomy and pancreatic divisum. Gastrointest Endosc 2015; 81: 1008 -1009

13 Sarhan MI, Choi JJ, Al Sawwaf M et al. Is weight loss better sustained with long-limb gastric bypass in the super-obese? Obes Surg 2011; 21: $1337-1343$

14 Saleem A, Levi MJ, Petersen BT et al. Laparoscopic assisted ERCP in Roux-en-Y gastric bypass (RYGB) surgery patients. J Gastrointest Surg 2012; 16: 203-208

15 Badaoui A, Malherbe $V$, Rosiere $A$ et al. ERCP by laparoscopic transgastric access and cholecystectomy at the same time in a patient with gastric bypass who was seen with choledocholithiasis. Gastrointest Endosc 2010; 71: 212-214

16 Peeters G, Himpens J. Laparoscopically assisted transgastric endoscopy in Roux-en-Y gastric bypass: a modification of the technique. Endoscopy 2009; 41: E190

17 Peeters G, Himpens J, Leman G. ERCP by laparoscopic transgastric access and cholecystectomy at the same time in a patient with gastric bypass who was seen with choledocholithiasis. Gastrointest Endosc 2010; 72: $1115-1116$

18 Tekola B, Wang AY, Ramanath $M$ et al. Percutaneous gastrostomy tube placement to perform transgastrostomy endoscopic retrograde cholangiopancreaticography in patients with Roux-en-Y anatomy. Dig Dis Sci 2011; 56: 3364-3369 